\title{
Evaluation of Mechanical Properties of $\Sigma 5(210) /[001]$ Tilt Grain Boundary with Self-Interstitial Atoms by Molecular Dynamics Simulation
}

\author{
Liang Zhang, ${ }^{1}$ Cheng Lu, ${ }^{1}$ Linqing Pei, ${ }^{1}$ Xing Zhao, ${ }^{2}$ Jie Zhang, ${ }^{1}$ and Kiet Tieu ${ }^{1}$ \\ ${ }^{1}$ School of Mechanical, Materials and Mechatronic Engineering, University of Wollongong, Wollongong, NSW 2522, Australia \\ ${ }^{2}$ State Key Laboratory of High Performance Complex Manufacturing, Central South University, Changsha 410083, China \\ Correspondence should be addressed to Cheng Lu; chenglu@uow.edu.au
}

Received 2 February 2017; Revised 10 May 2017; Accepted 30 May 2017; Published 20 July 2017

Academic Editor: Jean M. Greneche

Copyright ( 92017 Liang Zhang et al. This is an open access article distributed under the Creative Commons Attribution License, which permits unrestricted use, distribution, and reproduction in any medium, provided the original work is properly cited.

Grain boundary (GB) can serve as an efficient sink for radiation-induced defects, and therefore nanocrystalline materials containing a large fraction of grain boundaries have been shown to have improved radiation resistance compared with their polycrystalline counterparts. However, the mechanical properties of grain boundaries containing radiation-induced defects such as interstitials and vacancies are not well understood. In this study, we carried out molecular dynamics simulations with embedded-atom method (EAM) potential to investigate the interaction of $\Sigma 5(210) /[001]$ symmetric tilt GB in Cu with various amounts of self-interstitial atoms. The mechanical properties of the grain boundary were evaluated using a bicrystal model by applying shear deformation and uniaxial tension. Simulation results showed that GB migration and GB sliding were observed under shear deformation depending on the number of interstitial atoms that segregated on the boundary plane. Under uniaxial tension, the grain boundary became a weak place after absorbing self-interstitial atoms where dislocations and cracks were prone to nucleate.

\section{Introduction}

Materials under radiation of high-energy particles and severe plastic deformation will develop point defects or defect clusters, which may subsequently evolve into microstructural flaws, such as voids, stacking fault tetrahedra, dislocation loops, or solute segregation [1-6]. These defects and flaws not only deteriorate the physical properties of materials but also cause direct structural failure [7]. Compared with the conventional polycrystalline materials, nanocrystalline materials exhibit the superior property of radiation resistance due to the significantly decreased grain size and the increased volume fraction of grain boundary (GB) $[1,2,8,9]$. It has been demonstrated that grain boundary can act as an efficient sink for reducing the accumulation of radiation defects in nanocrystalline metals [10-13], alloys [14, 15], and oxides [16, 17]. For example, experiments showed that grain boundary could act as a sink for both self-interstitial atoms (SIAs) and vacancies, resulting in a denuded zone in the vicinity of an interface region [18]. Also, the increased radiation resistance has been confirmed in the early experiments of ion irradiation in nanocrystalline $\mathrm{Au}$ [10] and Pd [19]. The radiation tolerance of nanocrystalline materials strongly depends on the diffusion, segregation, and annihilation of SIAs and vacancies near the grain boundary. The fundamental processes have been extensively studied by atomistic simulations to examine the interaction of defects with grain boundary at the nanoscale [19-23]. Samaras et al. [19] conducted molecular dynamics (MD) simulation in nanocrystalline $\mathrm{Ni}$ to study the movement of collision cascade induced SIAs and their annihilation at the grain boundary. Bai et al. [3] used MD simulations to show that the grain boundary can absorb vacancies and interstitials through various mechanisms in $\mathrm{Cu}$. The efficient trapping of radiation-induced defects by the grain boundary contributes to the enhanced radiation resistance of nanocrystalline materials.

It is thermodynamically favorable for SIAs and vacancies to aggregate at the grain boundary, but the SIAs were found to be absorbed by the grain boundary more easily [3, 24-26]. Using molecular statics simulation at $0 \mathrm{~K}$, Tschopp et al. [25] 


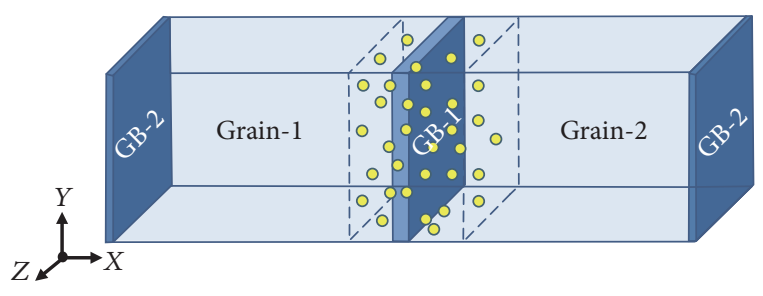

(a)

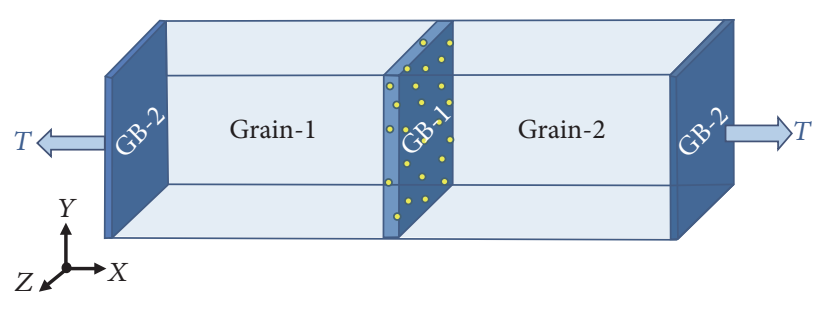

(c)

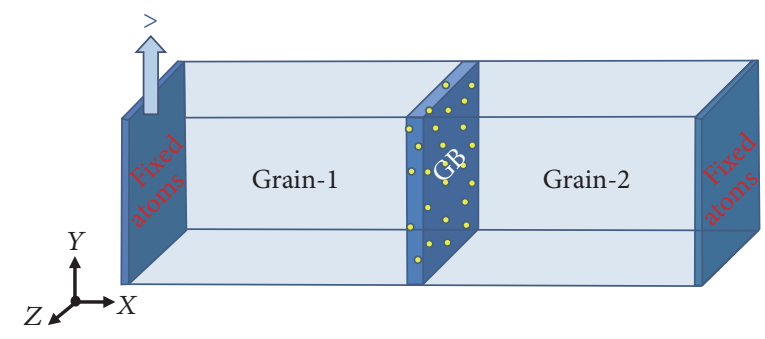

(b)

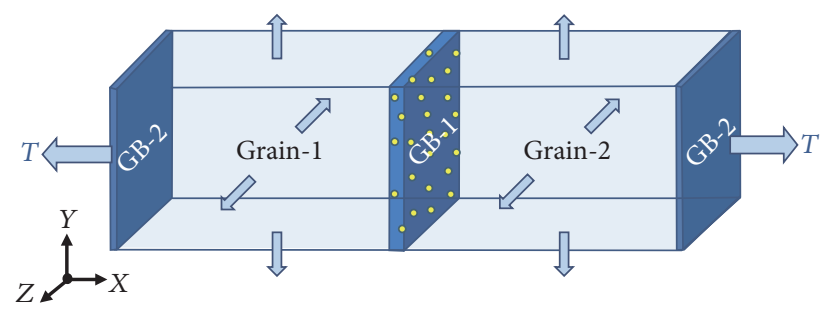

(d)

FIGURE 1: Schematic of the bicrystal model in different simulation cases. (a) Energy minimization and system relaxation. (b) Shear deformation. (c) Uniaxial tension under free boundary condition. (d) Uniaxial tension under constrained boundary condition. The yellow dots represent the introduced SIAs.

carried out extensive energetic calculations of the segregation of SIAs and vacancies in a-Fe. They found that SIAs have a larger energetic driving force for binding to grain boundaries than the vacancies. Li et al. $[27,28]$ reported similar results for the segregation of SIAs and vacancies in $\mathrm{Fe}, \mathrm{Mo}$, and W. In addition, MD simulations revealed that SIAs can be absorbed by the grain boundary at the early stage of radiation damage, whereas the vacancies were observed to be less mobile at the nanosecond timescale of the MD simulations [3, 24, 29-32]. While the role of the grain boundary as a defect sink has been extensively investigated in the previous research works, some recent studies have been focusing on the radiationinduced modification of GB structures and their influence on GB motion. For example, by using MD simulations, Frolov et al. $[33,34]$ investigated the structural transformation of $\mathrm{Cu}$ $\Sigma 5 \mathrm{~GB}$ by introducing point defects into the boundary region. Cheng et al. [35] and Zhang et al. [36] studied the effect of SIAs and vacancies on the shear-coupled GB motion. In these studies, the point defects introduced into the grain boundary were achieved by changing the position of the simulation grains, or by deleting and adding certain atoms at the GB region [35-37]. These methods cannot well represent the real situation under radiation, where the segregation of point defects at the grain boundary is an entirely random process. Until now, it is not very clear how the point defects randomly segregate at the grain boundary and what the subsequent strengthening and embrittling effects are. Studying this problem is beneficial to comprehensively understand the influence of radiation on the mechanical property of nanocrystalline materials.

In this work, we focused on studying the interaction between the grain boundary and SIAs by using MD simulations. On the one hand, SIAs are more prone to aggregate at the grain boundary than vacancies during displacement cascades. On the other hand, the subnanosecond timescale available to MD allows MD to address the evolution of the SIAs, but the motion of vacancies and their cluster is outside of the MD simulation time frame. Bicrystal models enable a well-controlled investigation of particular GB properties, and previous simulations took this advantage to study the mechanical properties of various GBs [38-41], and this approach was followed in the present study. The $\mathrm{Cu}$ $\Sigma 5(210) /[001] \mathrm{GB}$ was taken as an example in this study; this is a typical high-angle boundary with a misorientation angle of 53.1. The $\Sigma 5(210) \mathrm{GB}$ was chosen to be studied because its structure has been reported in many of the previous experiments and simulations. Firstly, we investigated the sink efficiency and the structural transformation of the $\Sigma 5(210)$ GB. Then, the shear and tensile response of the $\Sigma 5(210)$ GB with different levels of SIAs content were studied.

\section{Simulation Method}

Simulations were carried out by molecular dynamics code LAMMPS [42] using the embedded-atom method (EAM) potential provided by Mishin et al. for $\mathrm{Cu}$ [43]. The bicrystal model was created by constructing two different oriented crystal lattices and joining them together at a planar interface parallel to the $Y-Z$ plane along the $X$ direction, as shown in Figure 1(a). In the coincidence site lattice (CSL) notation, the $\Sigma 5[001](210)$ GB was generated through rotation of the left grain about the [001] tilt axis by $\theta / 2\left(\theta=53.1^{\circ}\right)$ in a clockwise direction and the right grain by $\theta / 2$ in an anticlockwise direction. The GB plane was the (210) crystallographic plane in the left grain and the $(2 \overline{1} 0)$ plane in the right grain. The dimensions of the simulation box along the $X, Y$, and $Z$ directions were set to about $242.5 \AA$, $97 \AA$, and $36.15 \AA$, respectively. The total number of atoms 

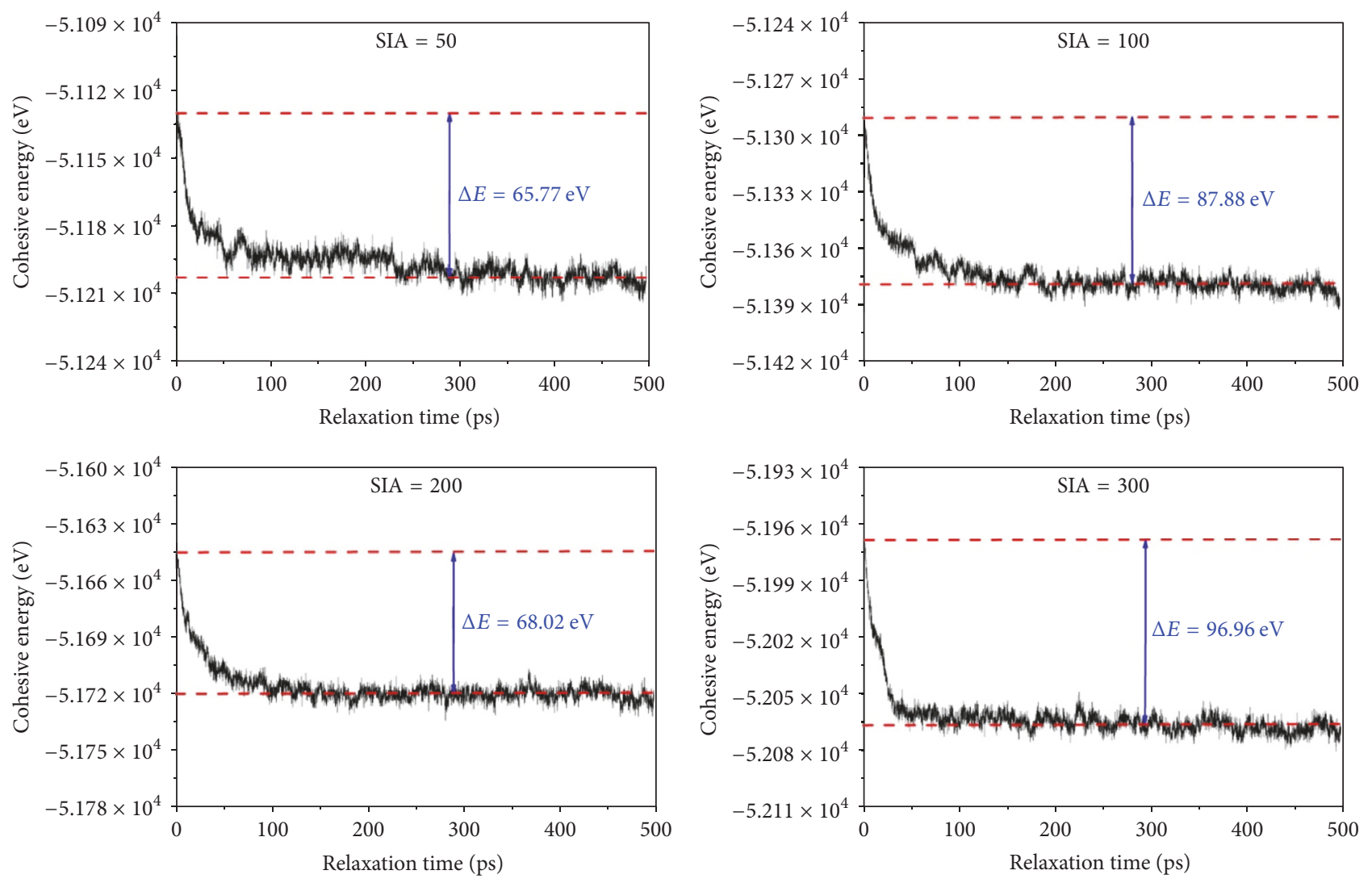

FIGURE 2: The cohesive energy of the GB area with various levels of SIA content as a function of system relaxation time.

in the bicrystal system was approximately $7.2 \times 10^{4}$. Periodic boundary conditions (PBC) were applied in all directions so that the isobaric-isothermal (NPT) ensemble can be used in all directions during system relaxation. The PBC applied in $Y$ and $Z$ directions simulate an infinite boundary plane between the two grains and can eliminate the effect of the free surface. The PBC in the $X$ direction introduced a second GB into the model, as indexed by GB-1 in the middle and GB-2 on the lateral sides. The SIAs were introduced by adding discrete $\mathrm{Cu}$ atoms in the area of $30 \AA$ width near GB-1, and they were randomly distributed on both sides of the boundary plane, as illustrated in Figure 1(a). The number of SIAs was set as $50,100,200$, and 300, respectively. The length of the bicrystal model $\left(L_{x}=242.5 \AA\right)$ can ensure that the introduced SIAs only interact with GB-1 and avoid any influence from GB-2, so that the effect of various levels of SIA content on the grain boundary behavior can be investigated.

The GB-SIAs interaction was simulated, and the relaxed GB structure was obtained by using molecular statics and the subsequent molecular dynamics simulation. The energy minimization procedure was used in the molecular statics calculation with a standard conjugate-gradient algorithm. Then, the bicrystal system was annealed at $300 \mathrm{~K}$ with a constant pressure of 0 bar in all directions for $500 \mathrm{ps}$. The time step was set as $1 \mathrm{fs}$ throughout the study. In the simulation case of SIA $=0$, the equilibrium GB structure was obtained after the energy minimization procedure, and the cohesive energy of the bicrystal system was stable during the annealing process. However, there is a significant decrease of the system energy in the simulation cases of the preexisting SIAs. It can be assumed that the relaxation of system energy was mainly due to the reduction of the total defects energy. For a clear illustration, we defined a GB area of $50 \AA$ width near the boundary plane in which the core structure of GB and all the nearby SIAs were included. Figure 2 plots the cohesive energy of the GB area as a function of annealing time at $300 \mathrm{~K}$ for SIA $=50,100,200$, and 300 , respectively. It was found that the cohesive energy dropped rapidly in the first $50 \mathrm{ps}$, and the value was convergent after $200 \mathrm{ps,} \mathrm{indicating}$ that the local equilibrium GB structure has been achieved. The red dashed line at the top indicates the energy value of the GB area at the initial stage of system relaxation, and the bottom one indicates the average energy value from 200 ps to 500 ps. The difference between the two dashed lines $(\Delta E)$ represents the reduction of the total defects energy. The calculated value of $\Delta E$ is $65.77 \mathrm{eV}, 87.88 \mathrm{eV}, 68.02 \mathrm{eV}$, and $96.96 \mathrm{eV}$, respectively. By visual inspection of the MD results, energy relaxation resulted from the segregation of SIAs into the grain boundary to achieve a lower energy position. The detailed atomic information will be presented in Section 3.1. After the simulation sample was fully relaxed after 500 ps, the shear deformation and uniaxial tension were applied, respectively, to investigate the mechanical response and the deformation mechanisms of the GB with various levels of SIA content; the simulation result will be presented in Sections 3.2 and 3.3. 


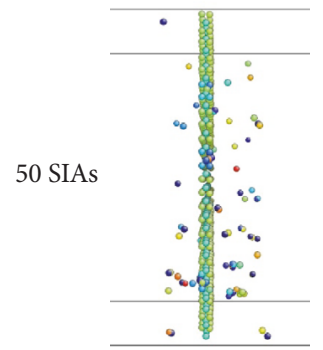

$m=0$ steps

(a1)

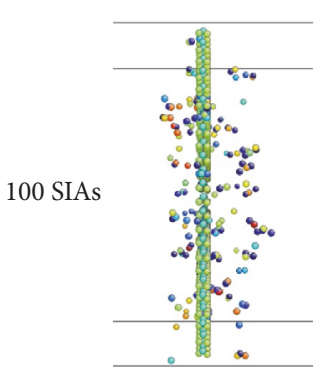

$m=0$ steps

(b1)

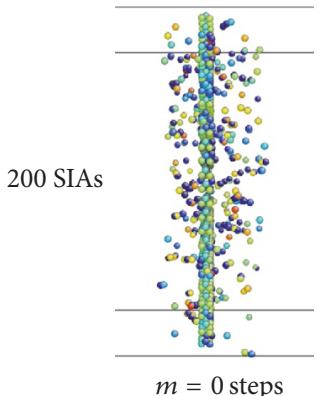

(c1)

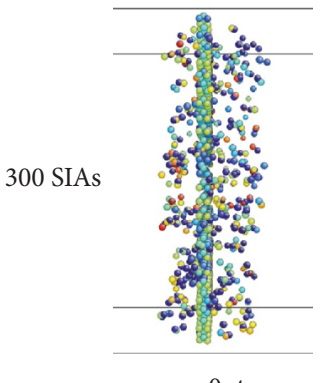

(d1)

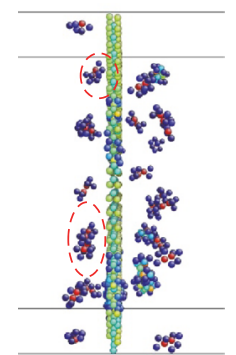

$m=820$ steps

(a2)

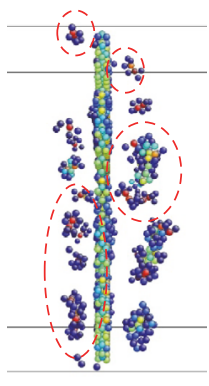

$m=1330$ steps

(b2)

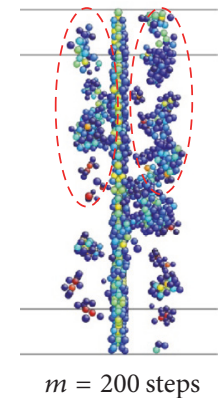

(c2)

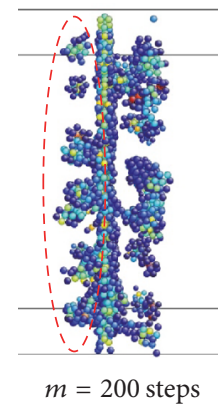

$(\mathrm{d} 2)$

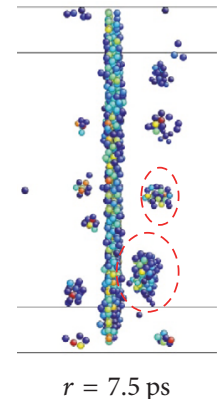

(a3)

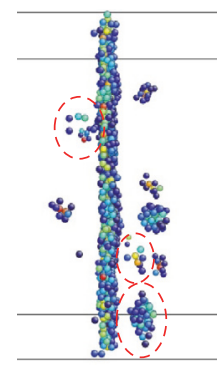

$r=10 \mathrm{ps}$

(b3)

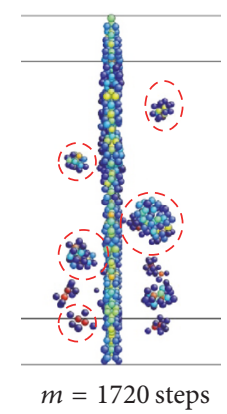

(c3)

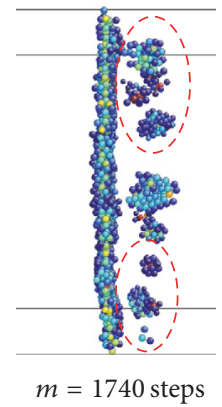

(d3)

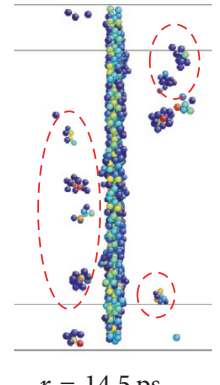

(a4)

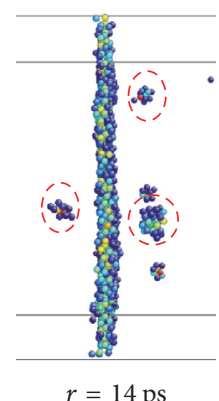

(b4)

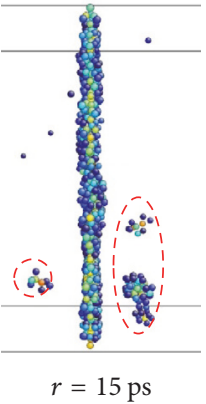

(c4)

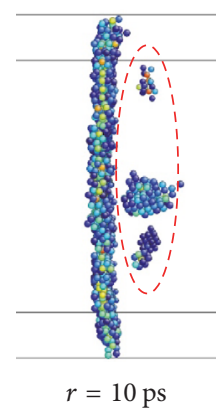

(d4)

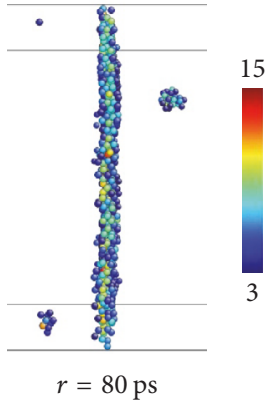

(a5)

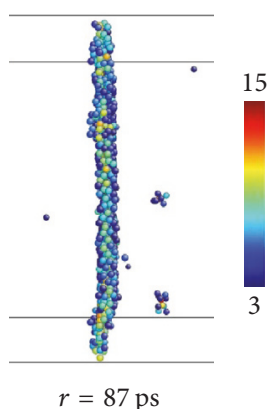

(b5)

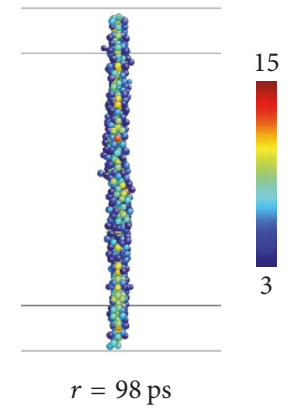

(c5)

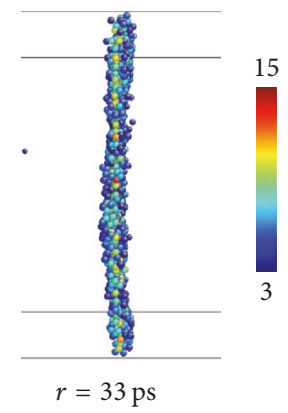

(d5)

FIGURE 3: Interaction of $\Sigma 5(210) /[001]$ GB with various levels of SIA content during the process of system energy minimization $(m)$ and the subsequent $300 \mathrm{~K}$ system relaxation $(r)$.

\section{Results and Discussion}

3.1. Interaction between $G B$ and SIAs. Figure 3 shows the snapshots of $\Sigma 5(210)$ GB with different numbers of SIAs during the process of system energy minimization (m) and the subsequent $300 \mathrm{~K}$ system relaxation (r). The initial GB configuration and the introduced SIAs are shown in Figures $3(\mathrm{al})-3(\mathrm{~d} 1)$. The atoms are colored according to the centrosymmetry value, and only the atoms with the value between 3 and 15 are shown to assist in the visualization of the 
GB and the SIA position. During the energy minimization, the single SIA tends to aggregate with the surrounding SIAs to form an SIA cluster, as shown in Figures 3(a2)-3(d2). The result agrees with the previous MD simulations that were carried out on single crystal structures where SIAs tend to cluster under cascade damage conditions [44, 45]. Compared to the vacancies, SIAs and their clusters have been seen to possess a much lower activation energy $[46,47]$ and therefore have a higher glissile mobility [48-50]. The rapid movement of SIAs was evidenced in the current study where the SIAs were prone to aggregate at the grain boundary even in the short timescale simulation. The energy minimization procedure was completed at 820 steps, 1330 steps, 1720 steps, and 1740 steps for the boundary with SIA $=50,100,200$, and 300, respectively. In Figures 3(c3) and 3(d3), it is interesting to find that the number of SIA clusters is less than that in Figures 3(a2) and 3(b2). The result indicates that some of the SIA clusters have already been absorbed by the GB during the energy minimization process in the cases of SIA $=200$ and SIA $=300$. In other words, the absorption of SIA by the grain boundary can occur without any thermodynamic assistance. The red dashed circles in Figure 3 highlight the SIA clusters which were annihilated in the next snapshot. Since there is a high density of interstitial atoms in the cases of SIA $=200$ and SIA $=300$, the result may imply that larger sized SIA clusters can be absorbed by the grain boundary more easily. This conclusion was consistent with the previous findings that the larger SIA clusters can show a higher mobility than the scattered point defects $[46,51]$. Most of the SIAs have been absorbed by the grain boundary after system relaxation for $50 \mathrm{ps}$ at $300 \mathrm{~K}$, resulting in the significant relaxation of the potential energy in the GB area. For the SIA $=200$ and SIA = $300 \mathrm{GBs}$, the preexisting SIAs have been completely absorbed by the grain boundary at $98 \mathrm{ps}$ and $33 \mathrm{ps}$, respectively, as shown in Figures 3(c5) and 3(d5). For the SIA $=50$ and SIA $=$ 100 GBs, a couple of SIAs or SIA clusters showed a random walk near the boundary plane and remained there after 100 ps system relaxation, as shown in Figures 3(a5) and 3(b5), but they were eventually absorbed by the boundary in the following annealing procedure. The equilibrium structure of $\mathrm{Cu} \Sigma 5(210) /[001] \mathrm{GB}$ can be described as consisting of a series of kite-shaped structural units, and each of the structural units contains six atoms [52]. However, the segregation of SIAs at GB can change its local structure significantly. For example, it was found that an extra atom exists in the midst of the six atoms in some structural units for SIA $=50 \mathrm{GBs}$, and this is also the case for other GBs with higher SIA content. The equilibrium structure is analogous to the SC-2 GB structure that was reported in our previous study on the $\Sigma 5(310) /[001]$ $\mathrm{GB}$ of $\mathrm{Al}[35]$.

3.2. Shear Deformation. In this section, shear stress was applied to investigate the mechanical response of the $\mathrm{Cu}$ bicrystal and the shear-induced GB motion at $300 \mathrm{~K}$. There is a slab of fixed atoms on the left-hand side and the righthand side of the simulation cell. The slab is approximately twice the cutoff radius of atomic interactions along the $X$ direction [39]. During the simulation, the fixed atoms were frozen in their perfect lattice positions while all the other atoms in the model were set free. A constant shear velocity $V_{s}$ $=0.5 \mathrm{~m} / \mathrm{s}$ parallel to the boundary plane was applied onto the fixed area of the left grain, as shown in Figure 1(b). The fixed atoms in the left grain do not participate in MD simulations and only move with $0.5 \mathrm{~m} / \mathrm{s}$ as a rigid body, while the fixed atoms in the right grain remained stationary. Stress and temperature calculations were performed on the dynamic atoms between the two fixed slabs. The stress tensor was calculated by the standard virial expression. MD simulations for shear loading were performed in the canonical NVT ensemble (Nose-Hoover thermostat) under a fixed volume. Figure 4 shows the shear stress and GB position as a function of the simulation time. The original GB position is defined as zero, and a negative value indicates that the boundary moved to the negative $X$ direction. The simulation result indicates that a "shear-coupled GB motion" occurred in all simulation cases (i.e., the shear stress applied parallel to the boundary plane can induce GB vertical migration). The shear-coupled GB motion can be quantified by the coupling factor $(\beta=$ $\left.V_{s} / V_{n}\right)$, where $V_{s}$ is the shear displacement and $V_{n}$ is the GB migration distance perpendicular to the boundary plane. The ideal $\beta$ value of a certain GB structure is a geometric constant that can be predicted based on the GB misorientation; for example, the ideal $\beta$ value of $\Sigma 5(210) /[001]$ GB was predicted as 0.667 [52]. The snapshots in Figure 4(a) show a typical migration process of the SIA $=0$ GBs at $t=0 \mathrm{~ns}$ and $t$ $=6 \mathrm{~ns}$ under shear stress at $300 \mathrm{~K}$. The atoms are colored according to the centrosymmetry value, and the atoms with perfect fcc environment are removed to facilitate the view of GB position. The sporadic atoms that scattered in the bicrystal model are due to the thermal disturbance of local atoms under shear stress. In Figure 4(b), the GB motion exhibits a "stick-slip" behavior (in the upper panel) and a "stop-and-go" character of motion (in the lower panel). The "stick-slip" behavior of the SIA $=0$ GBs is characterized by sawtooth time dependence of stress, and the "stop-andgo" movement is represented by continuous regular steps. When the GB is stationary ("stop" period), system stress builds up at a fixed rate until the accumulated stress is large enough to drive the boundary to move ("go" period). The GB jumps abruptly at the peak stress while the buildup stress is relieved to a lower level. This GB movement results in a plateau followed by a step in the GB position curve. The periodic process keeps driving the motion of the boundary to the negative $X$ direction. The average value of the peak stress in each migration cycle is $0.18 \mathrm{GPa}$ at $300 \mathrm{~K}$, and the calculated coupling factor is $\beta=0.678$ for SIA $=0$ GBs. The coupling factor from simulation agrees with the predicted $\beta$ value of the $\Sigma 5(210) /[001] \mathrm{GB}$, which indicates a perfect shear-coupled GB motion.

For the SIA $=50 \mathrm{GBs}$, the coupling factor was calculated as $\beta=0.676$, which also followed the perfect coupling GB motion. However, the critical shear stress required to activate the GB migration is $0.65 \mathrm{GPa}$, and the peak stress in each migration cycle reduces gradually to a convergent value $(\approx 0.25 \mathrm{GPa})$ with the progress of the shear strain, as shown in Figure 4(c). The GB trajectory shows that the introduced SIAs at the boundary resulted in a less regular "stop-and-go" motion of SIA $=50 \mathrm{GBs}$ than the SIA $=0 \mathrm{GBs}$, but the overall 


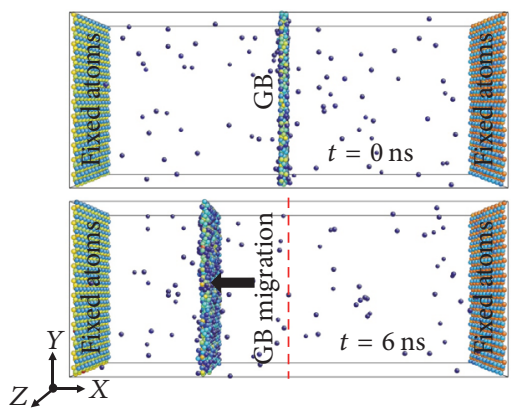

(a) SIA $=0$ GBs
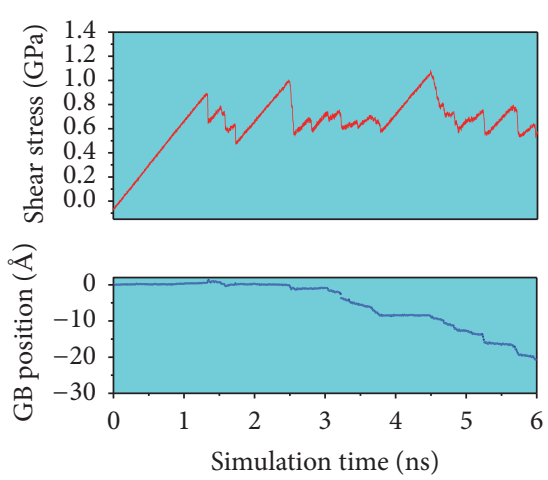

(d) SIA $=100 \mathrm{GBs}$

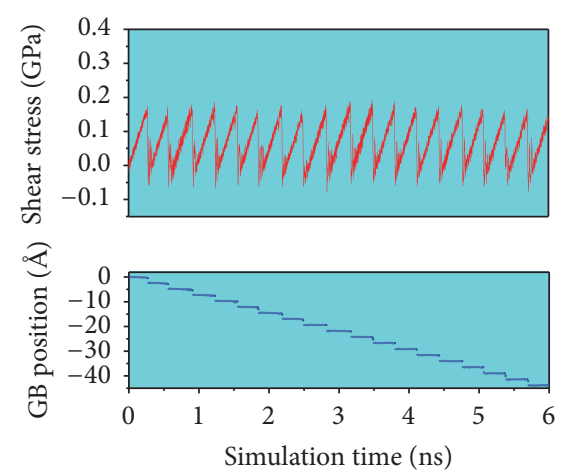

(b) SIA $=0 \mathrm{GBs}$
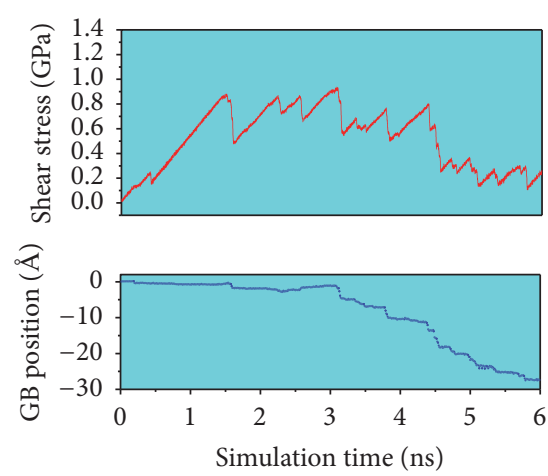

(e) SIA $=200 \mathrm{GBs}$

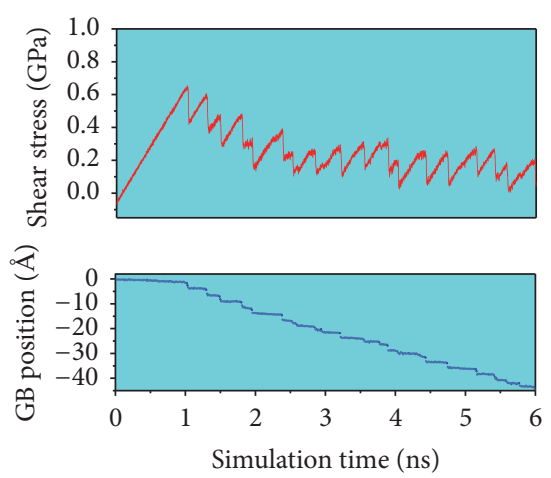

(c) $\mathrm{SIA}=5 \mathrm{GBs}$
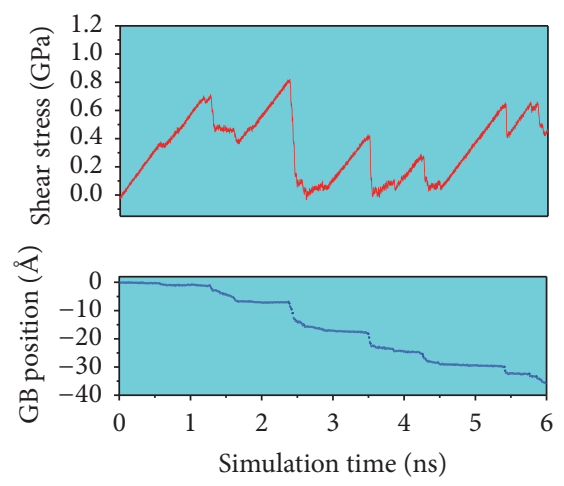

(f) $\mathrm{SIA}=300 \mathrm{GBs}$

FIGURE 4: Shear response of $\Sigma 5(210) /[001]$ GBs with various levels of SIA content at $300 \mathrm{~K}$. (a) Snapshots of the bicrystal model with SIA $=0$ GBs at $t=0 \mathrm{~ns}$ and $t=6 \mathrm{~ns}$. (b)-(f) Shear stress and the GB position as a function of simulation time with different numbers of SIAs.

shear-coupled GB motion has not been changed. By visual inspection of the MD results, it was found that the coupling mechanism was identical for the SIA $=0$ GBs and SIA $=50$ GBs; the shear-coupled GB migration was achieved by the collective motion of the structural units on the boundary plane [52]. The result of SIA $=50 \mathrm{GBs}$ reveals that a small number of SIAs that segregated into GB may not essentially change the deformation mechanism of GB under shear. For the SIA $=100$ GBs, the bicrystal model deformed elastically as shear strain was applied at the initial stage. However, the boundary structure became chaotic due to the higher level of the SIA contents, and the atoms involved in the structural units were shuffled. The first stress relaxation from $0.88 \mathrm{GPa}$ was associated with GB sliding, as shown in Figure 4(d); the GB was still at the starting position after several stress cycles. The GB sliding process lasts for about $3 \mathrm{~ns}$ followed by the GB migration process. A similar mechanism has been observed for SIA $=200 \mathrm{GBs}$ and SIA $=300 \mathrm{GBs}$. At the initial stage, the shear strain induces a distortion of the existing structure units and buildup elastic stresses in the surrounding crystal lattice. Recall that the SIAs that segregated into grain boundary were finally located at the core of the structural unit of the $55(210)$ GB. The higher contents of SIAs can change the local equilibrium structure more significantly so that the conventional migration mechanism of $\Sigma 5(210)$ GB is altered. It was supposed that a contradictory motion might arise if different GB structures coexist in one boundary plane because different moving behaviors could be triggered when subjected to shear stress. Therefore, when the local shear stress at the boundary reaches a critical level $(0.87 \mathrm{GPa}$ and $0.68 \mathrm{GPa}$, resp.), the uncoordinated transition of the structural units causes the disorder of local atoms and induces the GB sliding process. During the shear deformation, GB sliding accompanied by GB migration is the dominated deformation mechanism.

3.3. Uniaxial Tension. Tensile deformation was performed by considering both "free" and "constrained" tension boundary conditions [38]. A constant rate of $1 \times 10^{8} \mathrm{~s}^{-1}$ was applied perpendicular to the boundary plane (along the $X$-axis) at a temperature of $300 \mathrm{~K}$. The time increment of simulations was fixed at $1 \mathrm{fs}$. Under free tension boundary condition, the bicrystal model in the lateral directions was allowed to expand or contract during the deformation process, and the lateral stresses were kept free $\left(\sigma_{y y}=\sigma_{z z}=0\right)$. Under constrained tension boundary condition, the models were strained at a constant rate along the $X$-axis while keeping the model dimensions along the $Y$ - and $Z$-axis fixed $\left(\varepsilon_{y y}=\right.$ $\left.\varepsilon_{z z}=0\right)$. This boundary condition considers the lateral stress along the $Y$ - and $Z$-axis during the tensile deformation process. The schematics of tension simulation with "free" and "constrained" boundary conditions are shown in Figures 1(c) and 1(d), respectively. For the mechanical properties, system stress was attained by calculating the pressure of the entire 


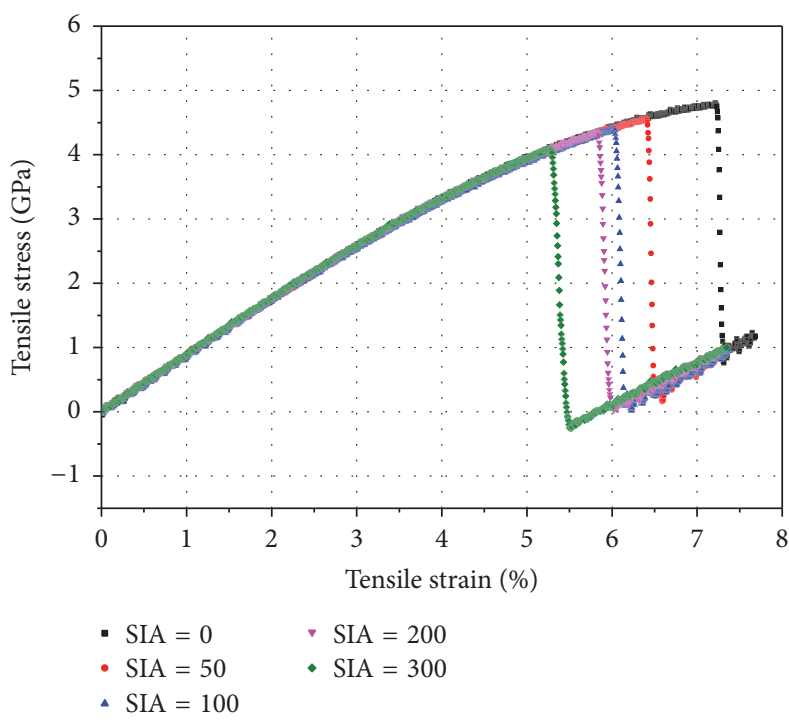

(a)

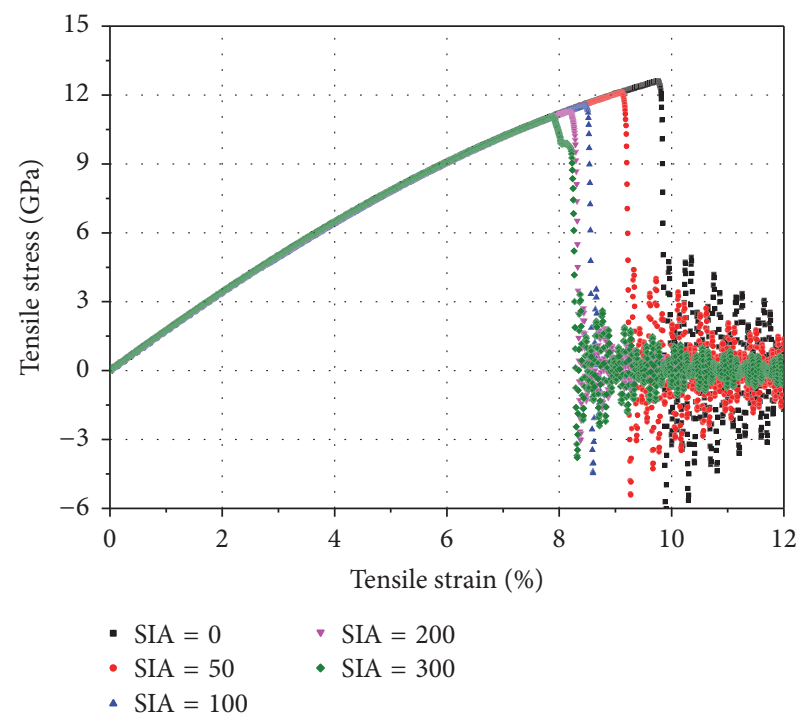

(b)

FIGURE 5: Tensile response of $\Sigma 5(210) /[001]$ GBs with various levels of SIA content at $300 \mathrm{~K}$. (a) Under free tension boundary condition. (b) Under constrained tension boundary condition.

system of atoms; system strain was derived from the positions of the periodic boundaries. Figure 5 shows the tensile stressstrain curves of $\Sigma 5(210) \mathrm{GB}$ with various levels of SIA content under two tension boundary conditions. In general, it was found that the maximum tensile stress decreased with the increasing number of SIAs at the boundaries. The different mechanical behavior of GBs can be associated with boundary energy, where the GB with a larger number of SIAs has higher boundary energy and becomes less stable to yield. Moreover, the maximum tensile stress obtained under the constrained boundary condition is significantly higher than the value from the free boundary condition. The higher tensile stress can be attributed to the stress that developed transverse to the loading direction during the deformation process.

Visual inspection of the MD simulation results indicates that the maximum tensile stress corresponds to the nucleation of partial dislocations from the boundary, in agreement with the results of our previous study on the symmetric and asymmetric $\mathrm{Cu} \Sigma 5$ GBs [38]. Figure 6 shows the atomic configurations of bicrystal that were captured at the incipient plastic stage under free tension boundary condition. Images were colored according to the centrosymmetry parameter, and atoms with perfect fcc structures were removed to facilitate viewing of the defective structures. At the initial stage of the stress drop in each simulation case, the image shows that dislocation loops with both edge and screw character were nucleated from GB-1. They are the Shockley partial dislocations with Burger's vectors $\mathbf{b}=(1 / 6)[112]$ and $\mathbf{b}=(1 / 6)[11 \overline{2}]$ and slip on the (111) and $(11 \overline{1})$ plane in Grain1 and Grain-2. The red dashed line indicates the dislocation cores followed by the stacking fault behind. Recall that the PBC applied on the $X$-axis introduced two identical GBs (GB-1 and GB-2), and our early studies showed that the dislocations nucleated simultaneously on both boundaries once the maximum stress has been achieved for the symmetric GBs
$[38,53,54]$. However, this is not the case for the current study, where dislocation nucleation was only evidenced from GB-1 that has been modified by absorbing SIAs. With the increase in the tensile strain, dislocations nucleate continuously from the GB plane, and their propagation and interactions contributed to the plastic deformation. Figure 7 shows the atomic configurations of bicrystal that were captured at the onset of plastic deformation under constrained tension boundary condition. Similarly, the decrease of the maximum tensile stress was associated with the nucleation of Shockley's partial dislocations from GB-1. Unlike the massive dislocations and their slipping in the bicrystal samples under free tension boundary condition, only limited dislocations were observed here. The nucleation of dislocation and its propagation have been restrained due to the lateral stress that was applied perpendicular to the tensile stress. Soon after the dislocations were nucleated, the crack initiated and extended along the GB plane in a brittle fracture manner, eventually causing the failure of the bicrystal samples. The cracks at the boundary plane were circled by dashed lines in Figure 7. Further progress of the tensile strain resulted in the cleavage of the GB-1 and the separation of the two grains.

\section{Remarks and Conclusions}

The above simulations have shown that the segregation of SIAs at the grain boundary can have a significant effect on its mechanical properties and deformation mechanisms. When the shear stress was applied, the deformation mechanism of $\Sigma 5(210)$ GB changed from the perfect shear-coupled motion to the combination of GB migration and GB sliding. Previous studies have shown that the GB sliding can only occur at a high temperature that approaches the premelting point for the symmetric $\Sigma 5$ GBs $[36,52]$. Therefore, the GB sliding event that was observed for $\Sigma 5(210) \mathrm{GB}$ with a high level of 


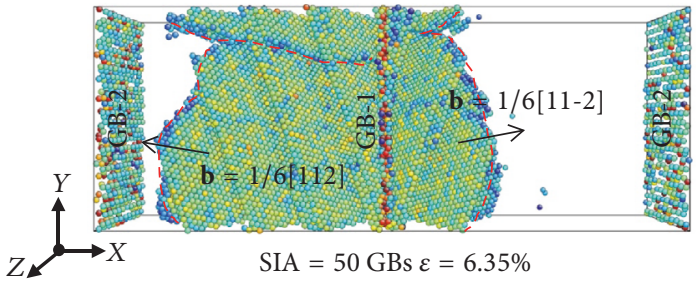

Centrosymmetry

(a)

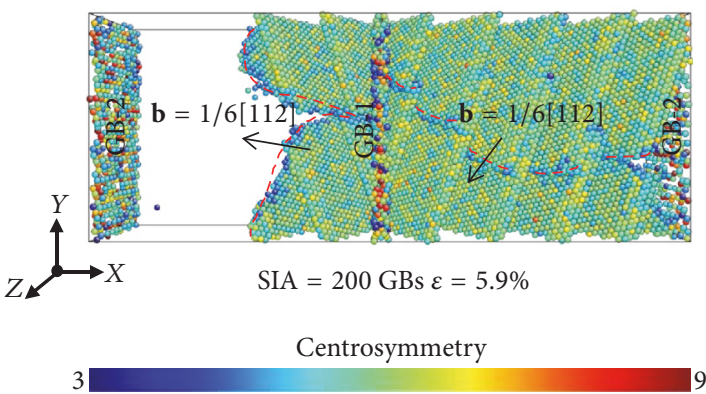

(c)

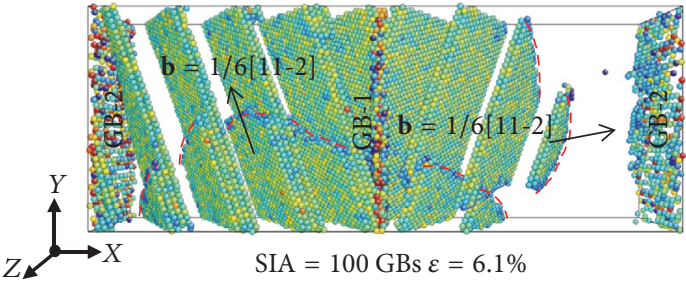

Centrosymmetry

(b)

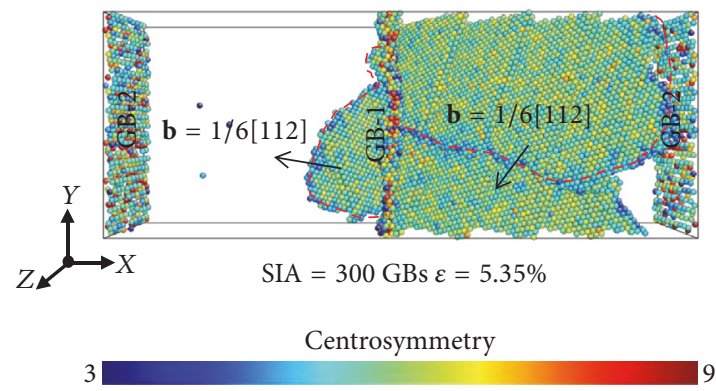

(d)

FIGURE 6: Snapshots of bicrystal samples under free tension boundary condition with (a) SIA = 50 GBs, (b) SIA = 100 GBs, (c) SIA =200 GBs, and (d) SIA $=300$ GBs.

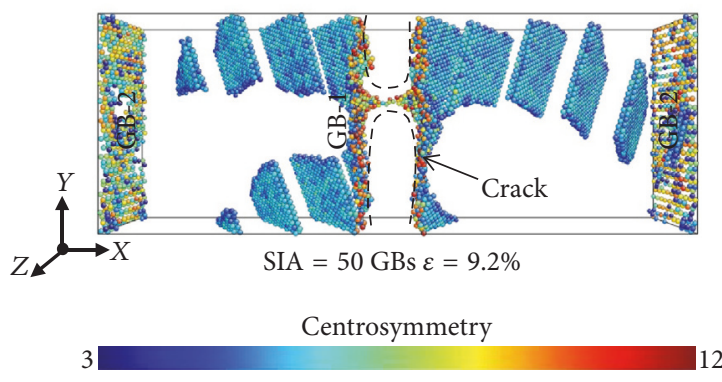

(a)

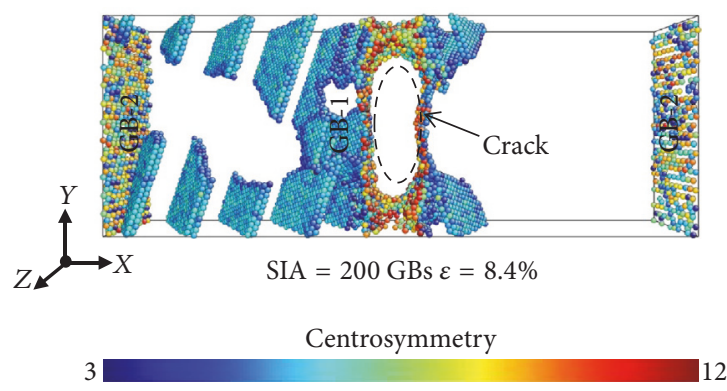

(c)

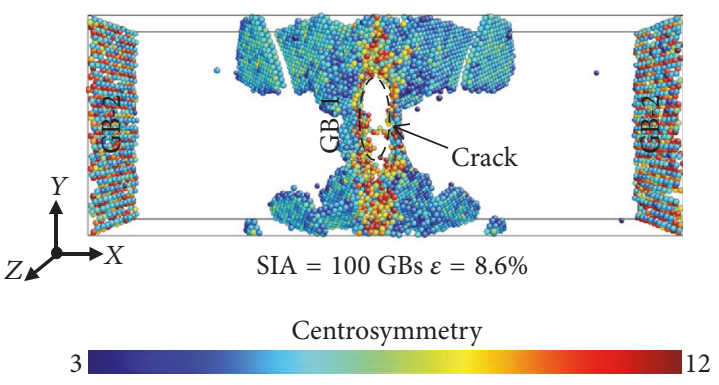

(b)

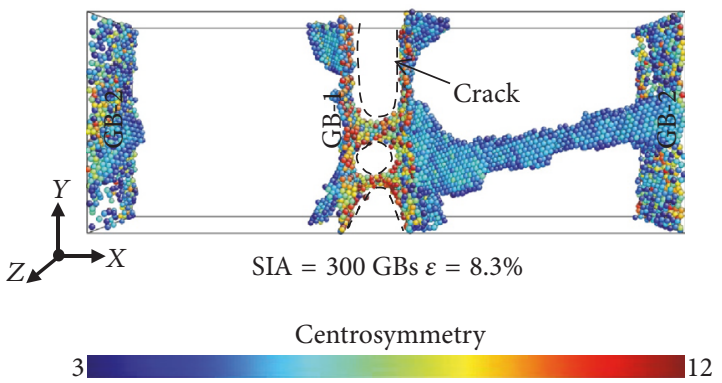

(d)

FIGURE 7: Snapshots of bicrystal samples under constrained tension boundary condition with (a) SIA = 50 GBs, (b) SIA = 100 GBs, (c) SIA = $200 \mathrm{GBs}$, and (d) SIA $=300 \mathrm{GBs}$.

SIA content at ambient temperature in return emphasized the role of SIAs and their associated change of local GB structure. The concept of "efficient temperature" $\left(T_{\text {eff }}\right)$ has been recently proposed for materials under severe irradiation [55] and severe plastic deformation $[56,57]$. According to this notion, if the atomic movements driven by deformation or irradiation were higher in comparison with the conventional thermal diffusion, the material could be forced to undergo a state which is equivalent to that at a certain increased (efficient) temperature $T_{\text {eff }}$. In this study, the segregation of SIAs could result 
in the phase transformation of the equilibrium structure of $\Sigma 5$ (210) GB, and therefore the behavior of $\Sigma 5(210)$ GB with high level segregated SIAs under shear deformation at ambient temperature $(T=300 \mathrm{~K})$ was equivalent to the GB performance at the premelting temperature $\left(T_{\text {eff }} \approx 700 \mathrm{~K}\right)$. Moreover, the notion of efficient temperature can be also applied to the simulation results of uniaxial tension. It is well known that the increased temperature can decrease the critical stress that is required to activate the dislocation nucleation and initiate the plastic deformation of materials. In this study, MD simulations indicated that the tensile strength of GB decreased with the increased level of SIA content under both free and constrained tensile boundary conditions. Therefore, one can expect that the efficient temperature that was required to initiate GB dislocation nucleation could be elevated with the increased amount of SIAs in a certain range. It is acknowledged that the limited length scales and the short timescales are the inherent limitations of the atomistic simulations at the current stage. Therefore, our study can only address the problem at the nanosecond timescale and high strain rates that are available to MD simulations. The high strain rates used in this work $\left(0.5 \mathrm{~m} / \mathrm{s}\right.$ in shear and $10^{8} / \mathrm{s}$ in tension), which are common to most MD simulations of inelastic behavior, may influence the dislocation nucleation process, but they have no significant effect on the main results of this study.

In conclusion, MD simulations were performed to investigate the mechanical response and deformation mechanisms of $\Sigma 5(210) /[001]$ GB in a Cu bicrystal with various levels of SIA content. The study of the interaction between $\Sigma 5(210)$ GB and the SIAs revealed that GB can play a role as an efficient sink to absorb interstitial atoms. The scattered SIAs were prone to aggregate to form SIA clusters, and the larger sized SIA clusters can be absorbed by the GB more easily. When shear deformation was applied parallel to the GB plane, a shear-coupled GB motion was observed. The coupling motion was perfect for the SIA $=0 \mathrm{GBs}$, and the coupling factor was consistent with the theoretical value. The introduced SIAs increased the critical stress that is required to activate the GB motion, and for the GBs with a high level of SIA content, GB migration was accompanied by the frequent GB sliding during shear deformation. Under uniaxial tension, the maximum tensile stress decreased with the increased number of SIAs. Dislocations nucleation and their propagation were the dominant mechanism under free tension boundary condition, while under constrained tension boundary condition, cracks were prone to initiate at the GB plane and resulted in a brittle mode of failure.

\section{Conflicts of Interest}

The authors declare that there are no conflicts of interest regarding the publication of this paper.

\section{Acknowledgments}

This work was supported by the Australia Research Council Discovery Project (DP170103092). Access to the high performance computer (HPC) facilities provided by the University of Wollongong is acknowledged.

\section{References}

[1] R. W. Grimes, R. J. M. Konings, and L. Edwards, "Greater tolerance for nuclear materials," Nature Materials, vol. 7, no. 9, pp. 683-685, 2008.

[2] G. Ackland, "Controlling radiation damage," Science, vol. 327, no. 5973, pp. 1587-1588, 2010.

[3] X.-M. Bai, A. F. Voter, R. G. Hoagland, M. Nastasi, and B. P. Uberuaga, "Efficient annealing of radiation damage near grain boundaries via interstitial emission," Science, vol. 327, no. 5973, pp. 1631-1634, 2010.

[4] V. Kuksenko, C. Pareige, and P. Pareige, "Intra granular precipitation and grain boundary segregation under neutron irradiation in a low purity Fe-Cr based alloy," Journal of Nuclear Materials, vol. 425, no. 1-3, pp. 125-129, 2012.

[5] K. Y. Yu, D. Bufford, C. Sun et al., "Removal of stacking-fault tetrahedra by twin boundaries in nanotwinned metals," Nature Communications, vol. 4, article no. 1377, 2013.

[6] L. Zhang, C. Lu, K. Tieu, L. Su, X. Zhao, and L. Pei, "Stacking fault tetrahedron induced plasticity in copper single crystal," Materials Science and Engineering: A, vol. 680, pp. 27-38, 2017.

[7] B. D. Wirth, "How does radiation damage materials?" Science, vol. 318, no. 5852, pp. 923-924, 2007.

[8] G. R. Odette, M. J. Alinger, and B. D. Wirth, "Recent developments in irradiation-resistant steels," Annual Review of Materials Research, vol. 38, pp. 471-503, 2008.

[9] S. J. Zinkle and N. M. Ghoniem, "Prospects for accelerated development of high performance structural materials," Journal of Nuclear Materials, vol. 417, no. 1-3, pp. 2-8, 2011.

[10] Y. Chimi, A. Iwase, N. Ishikawa, M. Kobiyama, T. Inami, and S. Okuda, "Accumulation and recovery of defects in ion-irradiated nanocrystalline gold," Journal of Nuclear Materials, vol. 297, no. 3, pp. 355-357, 2001.

[11] H. Kurishita, Y. Amano, S. Kobayashi et al., "Development of ultra-fine grained $\mathrm{W}-\mathrm{TiC}$ and their mechanical properties for fusion applications," Journal of Nuclear Materials, vol. 367-370, pp. 1453-1457, 2007.

[12] H. Kurishita, S. Kobayashi, K. Nakai et al., "Development of ultra-fine grained $\mathrm{W}-(0.25-0.8) \mathrm{wt} \% \mathrm{TiC}$ and its superior resistance to neutron and $3 \mathrm{MeV}$ He-ion irradiations," Journal of Nuclear Materials, vol. 377, no. 1, pp. 34-40, 2008.

[13] K. Y. Yu, Y. Liu, C. Sun et al., "Radiation damage in helium ion irradiated nanocrystalline Fe," Journal of Nuclear Materials, vol. 425, no. 1-3, pp. 140-146, 2012.

[14] D. A. McClintock, D. T. Hoelzer, M. A. Sokolov, and R. K. Nanstad, "Mechanical properties of neutron irradiated nanostructured ferritic alloy 14YWT," Journal of Nuclear Materials, vol. 386-388, no. C, pp. 307-311, 2009.

[15] A. Kilmametov, A. Balogh, M. Ghafari et al., "Radiation effects in bulk nanocrystalline FeAl alloy," Radiation Effects and Defects in Solids, vol. 167, no. 8, pp. 631-639, 2012.

[16] T. D. Shen, S. Feng, M. Tang, J. A. Valdez, Y. Wang, and K. E. Sickafus, "Enhanced radiation tolerance in nanocrystalline Mg Ga2 O4," Applied Physics Letters, vol. 90, no. 26, Article ID 263115, 2007.

[17] K. E. Sickafus, L. Minervini, R. W. Grimes et al., "Radiation tolerance of complex oxides," Science, vol. 289, no. 5480, pp. 748-751, 2000.

[18] B. N. Singh and S. J. Zinkle, "Defect accumulation in pure fcc metals in the transient regime: a review," Journal of Nuclear Materials, vol. 206, no. 2-3, pp. 212-229, 1993. 
[19] M. Samaras, P. M. Derlet, H. Van Swygenhoven, and M. Victoria, "SIA activity during irradiation of nanocrystalline Ni," Journal of Nuclear Materials, vol. 323, no. 2-3, pp. 213-219, 2003.

[20] D. Terentyev and X. He, "Dimensionality of interstitial He migration in <110> tilt grain boundaries in $\alpha$-Fe," Computational Materials Science, vol. 49, no. 4, pp. 858-864, 2010.

[21] F. Gao, H. Heinisch, and R. J. Kurtz, "Diffusion of He interstitials in grain boundaries in $\alpha$-Fe," Journal of Nuclear Materials, vol. 351, no. 1-3, pp. 133-140, 2006.

[22] F. Gao, H. L. Heinisch, and R. J. Kurtz, "Migration of vacancies, He interstitials and He-vacancy clusters at grain boundaries in $\alpha$-Fe," Journal of Nuclear Materials, vol.386-388, no. C, pp. 390394, 2009.

[23] R. J. Kurtz, H. L. Heinisch, and F. Gao, "Modeling of He-defect interactions in ferritic alloys for fusion," Journal of Nuclear Materials, vol. 382, no. 2-3, pp. 134-142, 2008.

[24] X.-M. Bai and B. P. Uberuaga, "The influence of grain boundaries on radiation-induced point defect production in materials: a review of atomistic studies," JOM, vol. 65, no. 3, pp. 360-373, 2013.

[25] M. A. Tschopp, K. N. Solanki, F. Gao, X. Sun, M. A. Khaleel, and M. F. Horstemeyer, "Probing grain boundary sink strength at the nanoscale: energetics and length scales of vacancy and interstitial absorption by grain boundaries in $\alpha$-fe," Physical Review B - Condensed Matter and Materials Physics, vol. 85, no. 6, Article ID 064108, 2012.

[26] X. Li, W. Liu, Y. Xu et al., "Radiation resistance of nanocrystalline iron: coupling of the fundamental segregation process and the annihilation of interstitials and vacancies near the grain boundaries," Acta Materialia, vol. 109, pp. 115-127, 2016.

[27] X. Li, W. Liu, Y. Xu et al., "Principal physical parameters characterizing the interactions between irradiation-induced point defects and several tilt symmetric grain boundaries in $\mathrm{Fe}$, Mo and W," Journal of Nuclear Materials, vol. 444, no. 1-3, pp. 229-236, 2014.

[28] X. Li, W. Liu, Y. Xu et al., "An energetic and kinetic perspective of the grain-boundary role in healing radiation damage in tungsten," Nuclear Fusion, vol. 53, no. 12, Article ID 123014, 2013.

[29] K. Sugio, Y. Shimomura, and T. D. De La Rubia, "Computer Simulation of Displacement Damage Cascade Formation near Sigma 5 Twist Boundary in Silver," Journal of the Physical Society of Japan, vol. 67, no. 3, pp. 882-889, 1998.

[30] F. J. Pérez Pérez and R. Smith, "Modelling radiation effects at grain boundaries in bcc iron," Nuclear Instruments and Methods in Physics Research, Section B: Beam Interactions with Materials and Atoms, vol. 153, no. 1-4, pp. 136-141, 1999.

[31] M. Samaras, P. M. Derlet, H. Van Swygenhoven, and M. Victoria, "Computer simulation of displacement cascades in nanocrystalline Ni," Physical Review Letters, vol. 88, no. 12, pp. 1255051-1255054, 2002.

[32] M. Samaras, P. M. Derlet, H. V. Swygenhoven, and M. Victoria, "Atomic scale modelling of the primary damage state of irradiated fcc and bcc nanocrystalline metals," Journal of Nuclear Materials, vol. 351, no. 1-3, pp. 47-55, 2006.

[33] T. Frolov, M. Asta, and Y. Mishin, "Segregation-induced phase transformations in grain boundaries," Physical Review, Article ID 020103, 2015.

[34] T. Frolov, D. L. Olmsted, M. Asta, and Y. Mishin, "Structural phase transformations in metallic grain boundaries," Nature Communications, vol. 4, article no. 1899, 2013.
[35] K. Cheng, L. Zhang, C. Lu, and K. Tieu, "Coupled grain boundary motion in aluminium: the effect of structural multiplicity," Scientific Reports, vol. 6, Article ID 25427, 2016.

[36] L. Zhang, C. Lu, G. Michal, K. Tieu, X. Zhao, and G. Deng, "Influence of temperature and local structure on the shearcoupled grain boundary migration," Physica Status Solidi (B) Basic Research, 2016.

[37] T. Frolov, "Effect of interfacial structural phase transitions on the coupled motion of grain boundaries: a molecular dynamics study," Applied Physics Letters, vol. 104, no. 21, Article ID 211905, 2014.

[38] L. Zhang, C. Lu, and K. Tieu, "Atomistic simulation of tensile deformation behavior of $\Sigma 5$ tilt grain boundaries in copper bicrystal," Scientific Reports, vol. 4, article no. 5919, 2014.

[39] L. Zhang, C. Lu, K. Tieu, X. Zhao, and L. Pei, "The shear response of copper bicrystals with $\Sigma 11$ symmetric and asymmetric tilt grain boundaries by molecular dynamics simulation," Nanoscale, vol. 7, no. 16, pp. 7224-7233, 2015.

[40] L. Zhang, C. Lu, and K. Tieu, "A review on atomistic simulation of grain boundary behaviors in face-centered cubic metals," Computational Materials Science, vol. 118, pp. 180-191, 2016.

[41] L. Zhang, C. Lu, J. Zhang, and K. Tieu, "A dual deformation mechanism of grain boundary at different stress stages," Materials Letters, vol. 167, pp. 278-283, 2016.

[42] S. Plimpton, "Fast parallel algorithms for short-range molecular dynamics," Journal of Computational Physics, vol. 117, no. 1, pp. $1-19,1995$.

[43] Y. Mishin, M. J. Mehl, D. A. Papaconstantopoulos, A. F. Voter, and J. D. Kress, "Structural stability and lattice defects in copper: $\mathrm{Ab}$ initio, tight-binding, and embedded-atom calculations," Physical Review B - Condensed Matter and Materials Physics, vol. 63, no. 22, Article ID 224106, pp. 2241061-22410616, 2001.

[44] C. H. Woo and B. N. Singh, "Production bias due to clustering of point defects in irradiation-induced cascades," Philosophical Magazine A: Physics of Condensed Matter, Structure, Defects and Mechanical Properties, vol. 65, no. 4, pp. 889-912, 1992.

[45] B. N. Singh and J. H. Evans, "Significant differences in defect accumulation behaviour between fcc and bcc crystals under cascade damage conditions," Journal of Nuclear Materials, vol. 226, no. 3, pp. 277-285, 1995.

[46] R. E. Stoller, G. R. Odette, and B. D. Wirth, "Primary damage formation in bcc iron," Journal of Nuclear Materials, vol. 251, pp. 49-60, 1997.

[47] P. Zhao and Y. Shimomura, "Molecular dynamics calculations of properties of the self-interstitials in copper and nickel," Computational Materials Science, vol. 14, no. 1-4, pp. 84-90, 1999.

[48] A. J. E. Foreman, C. A. English, and W. J. Phythian, "Molecular dynamics calculations of displacement threshold energies and replacement collision sequences in copper using a many-body potential," Philosophical Magazine A: Physics of Condensed Matter, Structure, Defects and Mechanical Properties, vol. 66, no. 5, pp. 655-669, 1992.

[49] H. Trinkaus, B. N. Singh, and A. J. E. Foreman, "Glide of interstitial loops produced under cascade damage conditions: Possible effects on void formation," Journal of Nuclear Materials, vol. 199, no. 1, pp. 1-5, 1992.

[50] H. Trinkaus, B. N. Singh, and A. J. E. Foreman, "Impact of glissile interstitial loop production in cascades on defect accumulation in the transient," Journal of Nuclear Materials, vol. 206, no. 2-3, pp. 200-211, 1993. 
[51] Y. N. Osetsky, A. Serra, and V. Priego, "Interactions between mobile dislocation loops in $\mathrm{Cu}$ and $\alpha$-Fe," Journal of Nuclear Materials, vol. 276, no. 1, pp. 202-212, 2000.

[52] J. W. Cahn, Y. Mishin, and A. Suzuki, "Coupling grain boundary motion to shear deformation," Acta Materialia, vol. 54, no. 19, pp. 4953-4975, 2006.

[53] L. Zhang, C. Lu, K. Tieu, L. Pei, X. Zhao, and K. Cheng, "Molecular dynamics study on the grain boundary dislocation source in nanocrystalline copper under tensile loading," Materials Research Express, vol. 2, no. 3, Article ID 035009, 2015.

[54] L. Zhang, C. Lu, K. Tieu, G. Michal, J. Zhang, and G. Deng, "Tension/compression asymmetry of grain boundaries with non-planar structure," Materials Research Express, vol. 3, no. 8, Article ID 085025, 2016.

[55] G. Martin, "Phase stability under irradiation: Ballistic effects," Physical Review B, vol. 30, no. 3, pp. 1424-1436, 1984.

[56] B. B. Straumal, A. A. Mazilkin, B. Baretzky, G. Schütz, E. Rabkin, and R. Z. Valiev, "Accelerated diffusion and phase transformations in $\mathrm{Co}-\mathrm{Cu}$ alloys driven by the severe plastic deformation," Materials Transactions, vol. 53, no. 1, pp. 63-71, 2012.

[57] B. Straumal, A. Korneva, and P. Zieba, "Phase transitions in metallic alloys driven by the high pressure torsion," Archives of Civil and Mechanical Engineering, vol. 14, no. 2, pp. 242-249, 2014. 

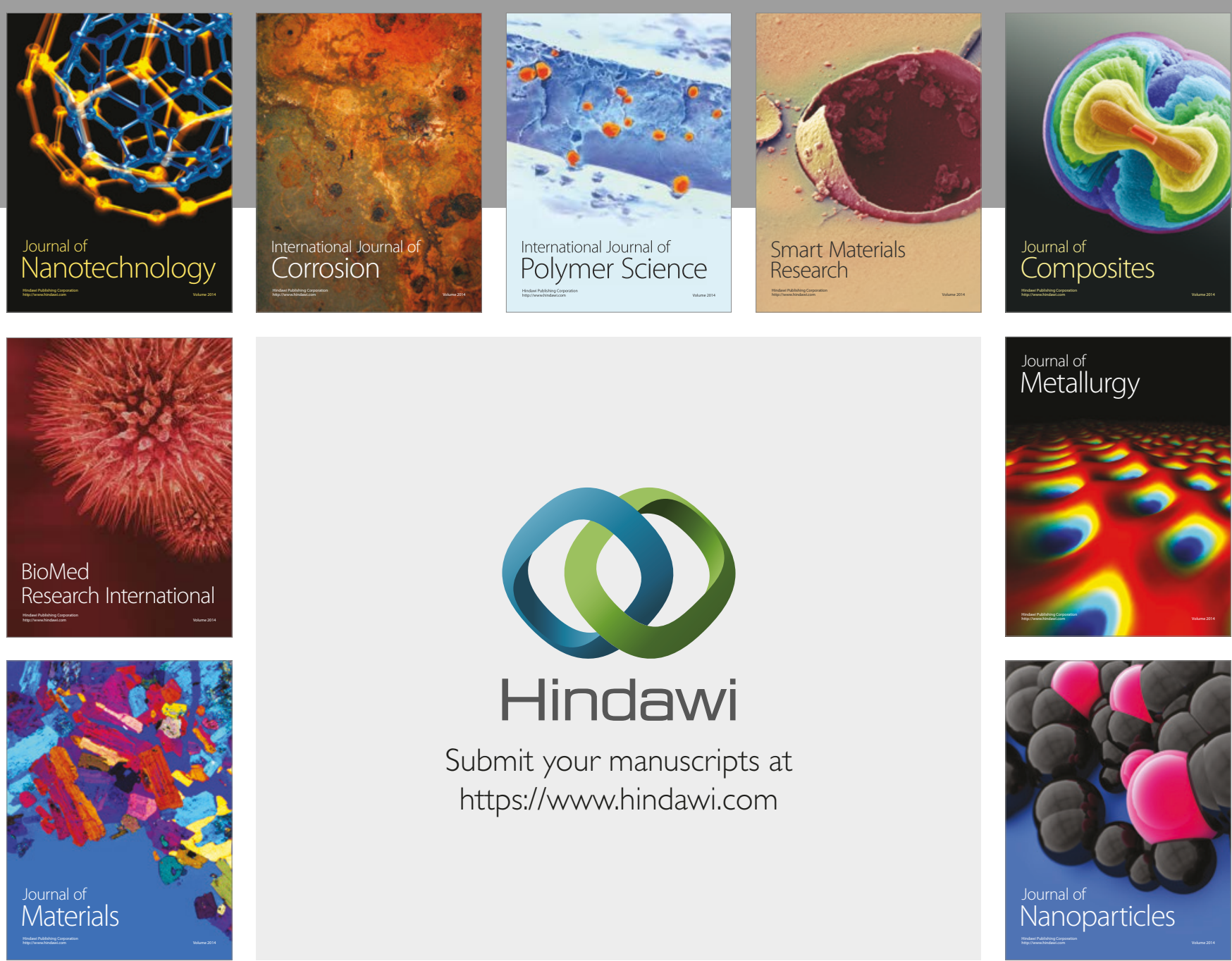

\section{Hindawi}

Submit your manuscripts at

https://www.hindawi.com
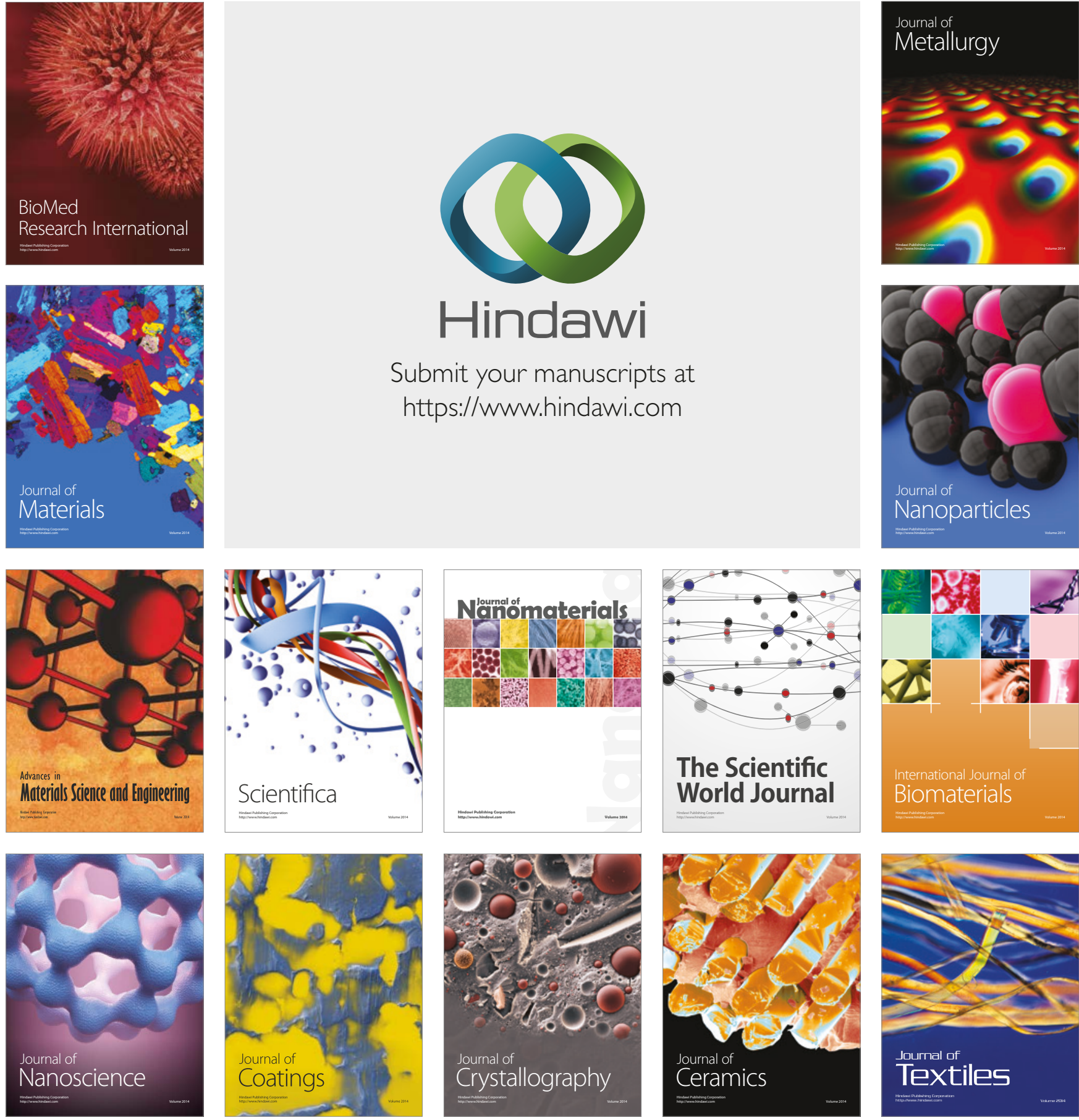

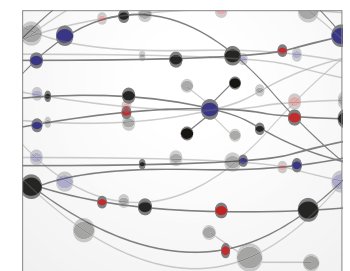

The Scientific World Journal
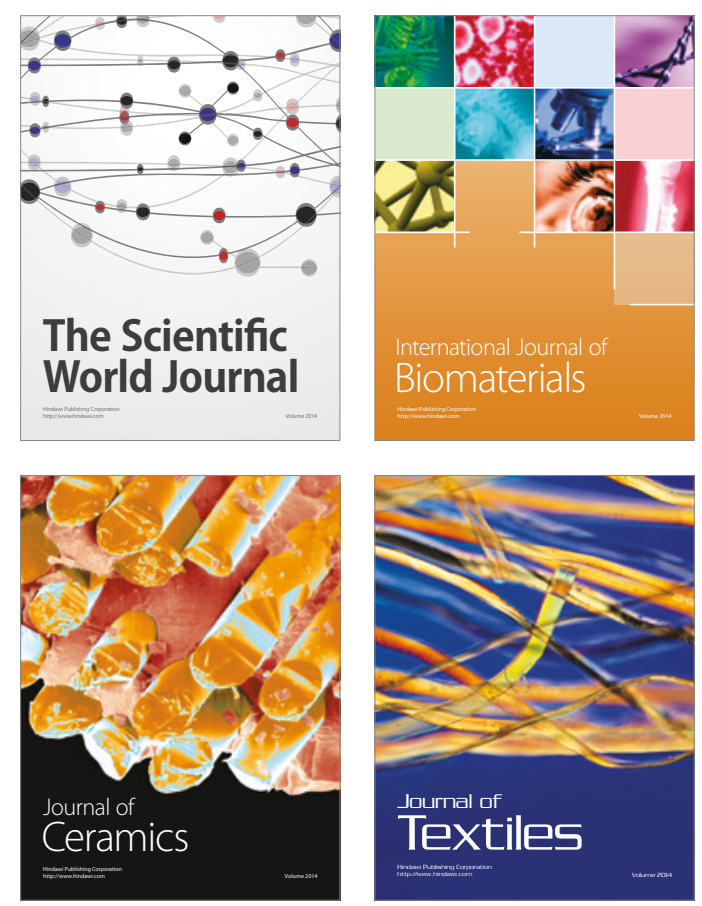